\title{
Analysis of Mathematics and Sustainability in an Impulsive Eutrophication Controlling System
}

\author{
Hengguo Yu, ${ }^{1,2}$ Min Zhao, ${ }^{2,3}$ and Qi Wang ${ }^{2,3}$ \\ ${ }^{1}$ School of Mathematics and Information Science, Wenzhou University, Wenzhou, Zhejiang 325035, China \\ ${ }^{2}$ Zhejiang Provincial Key Laboratory for Water Environment and Marine Biological Resources Protection, Wenzhou University, \\ Wenzhou, Zhejiang 325035, China \\ ${ }^{3}$ School of Life and Environmental Science, Wenzhou University, Wenzhou, Zhejiang 325035, China
}

Correspondence should be addressed to Min Zhao; zmcn@tom.com

Received 1 September 2013; Accepted 12 October 2013

Academic Editor: Luca Guerrini

Copyright (C) 2013 Hengguo Yu et al. This is an open access article distributed under the Creative Commons Attribution License, which permits unrestricted use, distribution, and reproduction in any medium, provided the original work is properly cited.

Eutrophication removal problems have captured the attention of biologists, mathematicians, and environmental scientists. Within this framework, an impulsive eutrophication controlling system is studied analytically and numerically. A key advantage of the eutrophication system is that it can be quite accurate to describe the interaction effect of some critical factors (fishermen catch and releasing small fry, etc.), which enables a systematic and logical procedure for fitting eutrophication mathematical system to real monitoring data and experiment data. Mathematical theoretical works have been pursuing the investigation of two threshold functions of some critical parameters under the condition of all species persistence, which can in turn provide a theoretical basis for the numerical simulation. Using numerical simulation works, we mainly focus on how to choose the best value of some critical parameters to ensure the sustainability of the eutrophication system so that the eutrophication removal process can be well developed with maximizing economic benefit. These results may be further extended to provide a basis for simulating the algal bloom in the laboratory and understanding the application of some impulsive controlling models about eutrophication removal problems.

\section{Introduction}

The eutrophication of lakes and reservoirs has been widely and intensively studied for several decades, which is a degradation process originating from introduction of nutrients from agricultural runoff and untreated industrial and urban discharges [1]. The eutrophication has been identified as a major and serious water quality management issue [2], not only in the developing countries [3-5], but also in the developed countries [6-8]. Impairment of water quality due to eutrophication can lead to a series of problems and result in losses of ecological integrity, sustainability, and safe use of aquatic ecosystems [9]. It is characterized by frequently recurring algal blooms, zooplankton aggregation, and reduction in species diversity in water bodies at all trophic levels [2].

In 1932, Bertalanffy firstly proposed a method of using mathematical model to study the biological system [10].
He pointed out that this approach is a combination of coordination, order, and purpose, which in turn formed three basic ideas of studying the biological system, namely, trophiclevel analysis, system perspective, and dynamic view. The main aim of modeling population dynamics is to improve the understanding of the interactions between populations and their dependence on internal and external conditions [11]. Hence, the mathematical models of biological population dynamics have to account not only for the growth and interactions between populations but also for the forecast of the disasters which can be caused by the plankton like blue-green algae, Moina and Brachionus. Early attempts began with the logistic growth, exponential growth, and Cui growth (this growth can explain the relationship between population increment and limiting resources) for biological population. With the increasing effects of the eutrophication, zooplankton aggregation, and algal blooms, the history of mathematical modeling plankton or zooplankton dynamics 
and biological eutrophication removal process is already quite long and has been initiated by biological science. These approaches have been refined to more realistic descriptions of the development of biological natural populations, especially algae population. In recent decades, the main researches focused on how to control the eutrophication, how to predict the outbreak of algae, how to simulate the spread tendency of algae, and how to develop eutrophication control strategy based on actual data. Hence, more and more researches have given an attempt to combine the forces of ecologist and mathematical engineers in an evaluation of possible control strategies for the management of eutrophication of water bodies, which is becoming an urgent watermanagement problem of drinking water reservoirs [12]. Malmaeus and Håkanson have depicted the development of a lake eutrophication model; they indicated that all changes that provided significant differences and improved predictions have been adopted [13]. Chen et al. said that their mathematical description provides a different insight into how to use nitrogen and phosphorus more effectively in the eutrophication control and elimination of blue-green blooms [14].

Zeya reservoir of Wenzhou is located in subtropical regions. Because of eutrophication, the nuisance algal blooms and the serious zooplankton aggregation frequently come forth, which caused clogging and blocking of the filtration system and resulted in millions of people without drinking water. Eutrophication removal is achieved by two major processes, physicochemical and biological treatment techniques; specifically biological eutrophication removal from drinking water in the lake and reservoir is usually considered to accomplish optimal and economic eutrophication treatment [15]. In order to understand and improve the performance of biological eutrophication removal treatment, it is necessary to briefly look into the mechanisms of eutrophication removal. The removal process of eutrophication in the Zeya reservoir is complex and dynamic with many variables. Since successful management of eutrophication in Zeya reservoir is often dependent on proper identification of the most likely growth limiting amount for algae, special emphasis is given to the limiting amount concept. In order to apply the biological principle to control eutrophication in the Zeya reservoir, we give good biological control agents for algae. First, we bring up two species of filter-feeding fish, silver carp (Hypophthalmichthys molitrix), and bighead carp (Aristichthys nobilis) [16], which are thought to be suited to control algae and zooplankton biomass directly in freshwater reservoir, especially cyanobacteria. Second, because the decision-maker must give cost-benefit and maintenance considerations, fish should be carried out and controlled at an appropriate time. At the same time, in order to prevent the rapid growth of plankton, especially algae, and maintain a good circulating development of biological process, we must release a certain amount of filter-feeding fish at a fixed time. These processes are subject to short-term perturbations whose durations are too small compared to the duration of the process. Consequently, it is natural to assume that these perturbations act instantaneously in the form of impulses. Thus impulsive differential equations and differential equations involving impulsive effects appear as a natural mathematical description (model) of observed evolution phenomena of several real world problems [17-21]. These works have introduced the theory of impulsive differential equations and its applications and provided a theoretical basis of the application of impulsive differential equations. In recent years, Fan et al. [22] have proposed the dynamics of a class of mutualismcompetition-predator interaction models with BeddingtonDeAngelis functional responses and impulsive perturbations; this work is important for solving the global stability and the globally exponential stability of system. Yu and Lu [23] mainly focused on the permanence and global attractivity of a discrete almost periodic ratio-dependent Leslie system with time delays and feedback controls; these works vigorously promote the application of feedback control. Shen et al. [24] mainly have studied the permanence of a single species system with distributed time delay and feedback controls; it is a very important fact that the feedback control is harmless to the permanence of species; these works are significant for the application of feedback control in biological control. Furthermore, the main aim of this investigation is to answer that what is the fundamental mathematical controlling mechanism, which can support the biological principle to control eutrophication in the Zeya reservoir and to provide a different insight into how to make the effects of environmental factors in connection with the ability of controlling eutrophication.

Speaking on eutrophication of Zeya reservoir, as the algae population grows to a certain biomass level, the dominant zooplankton species (Moina, Cyclops, and Brachionus are mainly grazing algae) are bound to sire and grow, so that these results can lead to damage or even crash of aquatic ecosystems. Furthermore, based on the above discussion, modeling is playing an increasing role in helping to identify causes and effective control measures through what if studies, and strategies are being identified for the management and control of eutrophication $[25,26]$; the paper considers an eutrophication mathematical model, which can be described by the following differential equations:

$$
\begin{gathered}
\frac{d x(t)}{d t}=r_{1} x(t)\left(1-\frac{x(t)}{k_{1}}\right)-\frac{a_{1} x(t) y(t)}{b_{1}+x(t)}-\frac{a_{2} x(t) z(t)}{b_{2}+x(t)}, \\
\frac{d y(t)}{d t}=r_{2} y(t)\left(1-\frac{y(t)}{k_{2}}\right)+\frac{e_{1} a_{1} x(t) y(t)}{b_{1}+x(t)}-\frac{a_{3} y(t) z(t)}{b_{3}+y(t)}, \\
\frac{d z(t)}{d t}=\frac{a_{2} e_{2} x(t) z(t)}{b_{2}+x(t)}+\frac{a_{3} e_{3} y(t) z(t)}{b_{3}+y(t)}-m z(t), \\
\Delta x(t)=-\delta_{1} x(t), \quad \Delta y(t)=-\delta_{2} y(t), \\
\Delta z(t)=-\delta_{3} z(t), \\
\quad t=(n+l-1) T, \\
\Delta x(t)=0, \quad \Delta y(t)=0, \quad \Delta z(t)=p, \quad t=n T
\end{gathered}
$$

where $x(t), y(t)$, and $z(t)$ are the biomass of the algae population (cyanobacteria) $\left(10^{6}\right.$ cell/L), the dominant zooplankton 
(Moina, Cyclops, and Brachionus) $\left(10^{2}\right.$ piece/L), and the filterfeeding fish (silver carp and bighead carp) (1 fish/Cubic meter) at time $t$, respectively, $\Delta x(t)=x\left(t^{+}\right)-x(t), \Delta y(t)=$ $y\left(t^{+}\right)-y(t)$, and $\Delta z(t)=z\left(t^{+}\right)-z(t) .0<l<1$ is used to describe the intervals of time between the pulsed use of controls. $r_{i}(i=1,2)$ are the intrinsic growth rate, $a_{i}(i=$ $1,2,3)$ and $b_{i}(i=1,2,3)$ measure the efficience of the prey in evading a predator attack. $e_{i}(i=1,2,3)$ denote the efficiency with which resources are converted to new consumers, $k_{i}$ $(i=1,2)$ are carrying capacity in the absence of the filterfeeding fish, and $m$ is the mortality rates for filter-feeding fish. The decision-maker of biological treatment technology must implement an impulsive control strategy that can better maintain biological treatment process, so we not only release a certain amount of the filter-feeding fishr $z(p>0$ is the release amount of the filter-feeding fishr $z$ ) at $t=n T$ to graze the algae and the dominant zooplankton, but also consider the algae and the dominant zooplankton losses due to other reasons as well as the filter-feeding fish harvest at $t=(n+l-$ 1)T $\left(\delta_{i}(i=1,2,3)\left(0<\delta_{i}<1\right)\right.$ represent the fraction of three species), where $T$ is the period of the impulsive effect, $n \in N$, and $N$ is the set of all nonnegative integers.

The main purpose of this paper is to enhance a theoretical and constructive framework by making the attempt to deal with eutrophication controlling problem for some drinking reservoirs using an impulsive eutrophication controlling system. Meanwhile, the theory discussions on the global asymptotical stability and permanent would help to design some efficient parameter constraint representations, which can in turn provide a theoretical basis for the numerical simulation. Further, in the context of population growth dynamics, how to implement impulsive control strategy to prevent and control the algal bloom is studied by simulating the dynamics of the impulsive controlling eutrophication system.

\section{Mathematical Analysis}

Now we will pursue the investigation of the constraint expression of the release amount $p$ and the average harvest $\delta_{3}$ of the filter-feeding fish. Let $R_{+}=[0, \infty)$, and $R_{+}^{3}=\{X \in$ $\left.R^{3} \mid X \geq 0\right\}$. Denote $f=\left(f_{1}, f_{2}, f_{3}\right)$ as the map defined by the right hand of the first, second, and third equations of system (1). Let $V: R_{+} \times R_{+}^{3} \rightarrow R_{+}$; then $V$ is said to belong to class $V_{0}$ if

(1) $V$ is continuous in $(n T,(n+l-1) T) \cup((n+l-$ 1)T, $(n+1) T] \times R_{+}^{3}$, and for each $X \in R_{+}^{3}, n \in$ $N, \lim _{(t, y) \rightarrow\left(n T^{+}, X\right)} V(t, y)=V\left(n T^{+}, X\right)$ exists;

(2) $V$ is locally Lipschitzian in $X$.

Definition 1. Let $V \in V_{0}$; then for $(t, x) \in(n T,(n+l-$ $1) T) \cup((n+l-1) T,(n+1) T] \times R_{+}^{3}$, the upper right derivative of $V(t, X)$ with respect to the impulsive differential system (1) is defined as

$$
\begin{aligned}
& D^{+} V(t, X) \\
& \quad=\lim _{h \rightarrow 0^{+}} \sup \frac{1}{h}[V(t+h, X+h f(t, x))-V(t, X)] .
\end{aligned}
$$

The solution of system (1) is a piecewise continuous function $X: R_{+} \rightarrow R_{+}^{3}, X(t)$ is continuous on $(n T,(n+$ $l-1) T) \cup((n+l-1) T,(n+1) T], n \in N$, and $X\left(n T^{+}\right)=$ $\lim _{t \rightarrow n T^{+}} X(t)$ exists. The smoothness properties of $f$ guarantee the global existence and uniqueness of solution of system (1); for details see $[8,9]$.

Definition 2. System (1) is said to be permanent if there exists a compact $\Omega \subset$ int $R_{+}^{3}$ such that every solution $(x(t), y(t), z(t))$ of system (1) will eventually enter and remain in the region $\Omega$.

The following lemma is obvious.

Lemma 3. Let $X(t)$ be a solution of system (1) with $X\left(0^{+}\right) \geq 0$; then $X(t) \geq 0$ for all $t \geq 0$, and further $X(t)>0, t>0$ if $X\left(0^{+}\right)>0$.

We will use an important comparison theorem on impulsive differential equation.

Lemma 4 (Bainov and Simeonov, 1993 [17]). Suppose $V \in V_{0}$. Assume that

$$
\begin{gathered}
D^{+} V(t, X) \leq g(t, V(t, X)), \quad t \neq n T, \quad t \neq(n+l-1) T, \\
V\left(t, X\left(t^{+}\right)\right) \leq \psi_{n}^{1}(V(t, X)), \quad t=(n+l-1) T, \\
V\left(t, X\left(t^{+}\right)\right) \leq \psi_{n}^{2}(V(t, X)), \quad t=n T,
\end{gathered}
$$

where $g: R_{+} \times R_{+}^{2} \rightarrow R_{+}^{2}$ is continuous in $((n-1) T,(n+l-1) T)$ and $((n+l-1) T, n T]$ for $u \in R_{+}^{2}, n \in N, \lim _{(t, v) \rightarrow\left(n T^{+}, u\right)} g(t, v)=$ $g\left(n T^{+}, u\right)$, and $\lim _{(t, v) \rightarrow\left((n+l-1) T^{+}, u\right)} g(t, v)=g\left((n+l-1) T^{+}, u\right)$ exists; $\psi_{n}^{i}(i=1,2): R_{+} \rightarrow R_{+}^{2}$ is nondecreasing. Let $r(t)$ be maximal solution of the scalar impulsive differential equation

$$
\begin{gathered}
\frac{d u(t)}{d t}=g(t, u(t)), \quad t \neq n T, \quad t \neq(n+l-1) T, \\
u\left(t^{+}\right)=\psi_{n}^{1}(u(t)), \quad t=(n+l-1) T, \\
u\left(t^{+}\right)=\psi_{n}^{2}(u(t)), \quad t=n T, \\
u\left(0^{+}\right)=u_{0},
\end{gathered}
$$

existing on $[0, \infty)$. Then $V\left(0^{+}, X_{0}\right) \leq u_{0}$ implies that $V(t, X(t)) \leq r(t), t \geq 0$, where $X(t)$ is any solution of system (1).

Finally, we give some basic properties about the following subsystems of system (1):

$$
\begin{gathered}
\frac{d z(t)}{d t}=-m z(t), \quad t \neq n T, t \neq(n+l-1) T \\
z\left(t^{+}\right)=\left(1-\delta_{3}\right) z(t), \quad t=(n+l-1) T \\
z\left(t^{+}\right)=z(t)+p, \quad t=n T \\
\left(0^{+}\right)=z_{0} .
\end{gathered}
$$


Clearly

$$
z^{*}(t)=\left\{\begin{array}{c}
\frac{p \exp (-m(t-(n-1) T))}{1-\left(1-\delta_{3}\right) \exp (-m T)} \\
(n-1) T<t \leq(n+l-1) T, \\
\frac{p\left(1-\delta_{3}\right) \exp (-m(t-(n-1) T))}{1-\left(1-\delta_{3}\right) \exp (-m T)} \\
(n+l-1) T<t \leq n T,
\end{array}\right.
$$

$n \in N$, and

$$
\begin{gathered}
z^{*}\left(0^{+}\right)=z^{*}\left(n T^{+}\right)=\frac{p}{1-\left(1-\delta_{3}\right) \exp (-m T)}, \\
z^{*}\left(l T^{+}\right)=\frac{p\left(1-\delta_{3}\right) \exp (-m l T)}{1-\left(1-\delta_{3}\right) \exp (-m T)}
\end{gathered}
$$

is a positive periodic solution of system (5). Since

$$
z(t)=\left\{\begin{array}{l}
\left(1-\delta_{3}\right)^{n-1}\left(z\left(0^{+}\right)-\frac{p}{1-\left(1-\delta_{3}\right) \exp (-m T)}\right) \\
\quad \times \exp (-m t)+z^{*}(t) \\
(n-1) T<t \leq(n+l-1) T \\
\left(1-\delta_{3}\right)^{n}\left(z\left(0^{+}\right)-\frac{p}{1-\left(1-\delta_{3}\right) \exp (-m T)}\right) \\
\quad \times \exp (-m t)+z^{*}(t) \\
(n+l-1) T<t \leq n T
\end{array}\right.
$$

is the solution of system (5) with initial value $z_{0}=z\left(0^{+}\right) \geq 0$, where $t \in((n-1) T,(n+l-1) T) \cup((n+l-1) T, n T], n \in N$, we get the following.

Lemma 5. For a positive periodic solution $z^{*}(t)$ of system (5) and every solution $z(t)$ of system (5) with $z_{0} \geq 0$, one has $\mid z(t)-$ $z^{*}(t) \mid \rightarrow 0, t \rightarrow \infty$.

Lemma 6. There exists a constant $M$ such that $x(t) \leq$ $M, y(t) \leq M, z(t) \leq M$, and $p(t) \leq M$ for each solution $(x(t), y(t), z(t), p(t))$ of system (1) with all $t$ largest enough.

Therefore, we obtain the complete expression for a periodic solution $\left(0,0, z^{*}(t)\right)$ of system (1). Now, we study the stability of the periodic solution, which explains the threshold expression of the release amount $p$ and the average harvest $\delta_{3}$ of the filter-feeding fish under the condition of some species extinction.
Theorem 7. Let $(x(t), y(t), z(t))$ be any solution of system (1); then $\left(0,0, z^{*}(t)\right)$ is said to be globally asymptotically stable if

$$
\begin{aligned}
r_{1} T & -\frac{a_{2}}{b_{2} m} \frac{p}{1-\left(1-\delta_{3}\right) \exp (-m T)} \\
& \times\left(1-\delta_{3} \exp (-m l T)-\left(1-\delta_{3}\right) \exp (-m T)\right) \\
< & \ln \left(\frac{1}{1-\delta_{1}}\right), \\
r_{2} T- & \frac{a_{3}}{b_{3} m} \frac{p}{1-\left(1-\delta_{3}\right) \exp (-m T)} \\
& \times\left(1-\delta_{3} \exp (-m l T)-\left(1-\delta_{3}\right) \exp (-m T)\right) \\
< & \ln \left(\frac{1}{1-\delta_{2}}\right) .
\end{aligned}
$$

Proof. The local stability of periodic solution $\left(0,0, z^{*}(t)\right)$ may be determined by considering the behavior of small amplitude perturbation of the solution. Define

$$
x(t)=u(t), \quad y(t)=v(t), \quad z(t)=w(t)+z^{*}(t) .
$$

We will put (10) into (1). The linearization of the system becomes

$$
\begin{gathered}
\frac{d u(t)}{d t}=\left(r_{1}-\frac{a_{2} z^{*}(t)}{b_{2}}\right) u(t), \\
\frac{d v(t)}{d t}=\left(r_{2}-\frac{a_{3} z^{*}(t)}{b_{3}}\right) v(t) \\
\frac{d w(t)}{d t}=-m w(t), \\
t \neq n T, \quad t \neq(n+l-1) T, \\
\Delta u(t)=-\delta_{1} u(t), \quad \Delta v(t)=-\delta_{2} v(t), \\
\Delta w(t)=-\delta_{3} w(t), \\
t=(n+l-1) T, \\
\Delta u(t)=0, \quad \Delta v(t)=0, \quad \Delta w(t)=0, \quad t=n T .
\end{gathered}
$$

Therefore, we have

$$
\left(\begin{array}{c}
u(t) \\
v(t) \\
w(t)
\end{array}\right)=\Phi(t)\left(\begin{array}{c}
u(0) \\
v(0) \\
w(0)
\end{array}\right), \quad 0 \leq t<T
$$

where $\Phi(t)$ satisfies

$$
\frac{d \Phi}{d t}=\left(\begin{array}{ccc}
r_{1}-\frac{a_{2} z^{*}(t)}{b_{2}} & 0 & 0 \\
0 & r_{2}-\frac{a_{3} z^{*}(t)}{b_{3}} & 0 \\
0 & 0 & -m
\end{array}\right) \Phi(t)
$$


and $\Phi(0)=I$, the identity matrix, and

$$
\begin{aligned}
&\left(\begin{array}{c}
u\left((n+l-1) T^{+}\right) \\
v\left((n+l-1) T^{+}\right) \\
w\left((n+l-1) T^{+}\right)
\end{array}\right) \\
& \quad=\left(\begin{array}{ccc}
1-\delta_{1} & 0 & 0 \\
0 & 1-\delta_{2} & 0 \\
0 & 0 & 1-\delta_{3}
\end{array}\right)\left(\begin{array}{c}
u((n+l-1) T) \\
v((n+l-1) T) \\
w((n+l-1) T)
\end{array}\right), \\
&\left(\begin{array}{c}
u\left(n T^{+}\right) \\
v\left(n T^{+}\right) \\
w\left(n T^{+}\right)
\end{array}\right)=\left(\begin{array}{lll}
1 & 0 & 0 \\
0 & 1 & 0 \\
0 & 0 & 1
\end{array}\right)\left(\begin{array}{c}
u(n T) \\
v(n T) \\
w(n T)
\end{array}\right) .
\end{aligned}
$$

The stability of the periodic solution $\left(0,0, z^{*}(t)\right)$ is determined by the eigenvalues of

$$
\Theta=\left(\begin{array}{ccc}
1-\delta_{1} & 0 & 0 \\
0 & 1-\delta_{2} & 0 \\
0 & 0 & 1-\delta_{3}
\end{array}\right)\left(\begin{array}{lll}
1 & 0 & 0 \\
0 & 1 & 0 \\
0 & 0 & 1
\end{array}\right) \Phi(T)
$$

If each of these eigenvalues is less than one in magnitude, then the periodic solution $\left(0,0, z^{*}(t)\right)$ is locally stable. Therefore all eigenvalues of $\Theta$, namely,

$$
\begin{gathered}
u_{1}=\left(1-\delta_{1}\right) \exp \left(\int_{0}^{T}\left(r_{1}-\frac{a_{2} z^{*}(t)}{b_{2}}\right) d t\right), \\
u_{2}=\left(1-\delta_{2}\right) \exp \left(\int_{0}^{T}\left(r_{2}-\frac{a_{3} z^{*}(t)}{b_{3}}\right) d t\right), \\
u_{3}=\left(1-\delta_{3}\right) \exp (-m T)<1 .
\end{gathered}
$$

According to Floquet theory of impulsive differential equation, $\left(0,0, z^{*}(t)\right)$ is locally asymptotically stable if $\left|u_{1}\right|<$ 1 and $\left|u_{2}\right|<1$; that is to say,

$$
\begin{aligned}
r_{1} T & -\frac{a_{2}}{b_{2} m} \frac{p}{1-\left(1-\delta_{3}\right) \exp (-m T)} \\
& \times\left(1-\delta_{3} \exp (-m l T)-\left(1-\delta_{3}\right) \exp (-m T)\right) \\
< & \ln \left(\frac{1}{1-\delta_{1}}\right), \\
r_{2} T- & \frac{a_{3}}{b_{3} m} \frac{p}{1-\left(1-\delta_{3}\right) \exp (-m T)} \\
& \times\left(1-\delta_{3} \exp (-m l T)-\left(1-\delta_{3}\right) \exp (-m T)\right) \\
< & \ln \left(\frac{1}{1-\delta_{2}}\right) .
\end{aligned}
$$

In the following, we will prove the global attraction. Choose a $\varepsilon>0$ such that

$$
\xi_{1} \triangleq\left(1-\delta_{1}\right) \exp \left(\int_{0}^{T}\left(r_{1}-\frac{a_{2}\left(z^{*}(t)-\epsilon\right)}{b_{2}+M}\right) d t\right)<1 .
$$

Note that $d z(t) / d t \geq-m z(t)$; from Lemma 5 and comparison theorem of impulsive equation, we have

$$
z(t)>z^{*}(t)-\epsilon
$$

for all $t$ large enough. For simplification, we may assume that (19) holds for all $t \geq 0$. From (1) and (19), we can obtain

$$
\begin{gathered}
\frac{d x(t)}{d t} \leq\left(r_{1}-\frac{a_{2}\left(z^{*}(t)-\epsilon\right)}{b_{2}+M}\right) x(t), \quad t \neq(n+l-1) T, \\
x\left(t^{+}\right)=\left(1-\delta_{1}\right) x(t), \quad t=(n+l-1) T,
\end{gathered}
$$

which leads to

$$
\begin{aligned}
x((n+l) T) \leq x\left((n+l-1) T^{+}\right) \\
\quad \times \exp \left(\int_{(n+l-1) T}^{(n+l) T}\left(r_{1}-\frac{a_{2}\left(z^{*}(t)-\epsilon\right)}{b_{2}+M}\right) d t\right) \\
=x((n+l-1) T)\left(1-\delta_{1}\right) \\
\quad \times \exp \left(\int_{(n+l-1) T}^{(n+l) T}\left(r_{1}-\frac{a_{2}\left(z^{*}(t)-\epsilon\right)}{b_{2}+M}\right) d t\right) \\
=x((n+l-1) T) \xi_{1} .
\end{aligned}
$$

Hence, $x((n+l) T) \leq x(l T) \xi_{1}^{n}$ and $x((n+l) T) \rightarrow 0$ as $n \rightarrow \infty$. Therefore, $x(t) \rightarrow 0$ as $n \rightarrow \infty$ since $0<x(t)<$ $x((n+l-1) T)\left(1-\delta_{1}\right) \exp \left(r_{1} T\right)$ for $(n+l-1) T<t \leq(n+l) T$. Similarly, $y(t) \rightarrow 0$ as $n \rightarrow \infty$.

Next, we prove that $z(t) \rightarrow z^{*}(t)$ as $t \rightarrow \infty$. For $0<\epsilon$ sufficiently small, there must exist a $T^{\prime}>0$ such that $0<$ $x(t)<\epsilon$ and $0<y(t)<\epsilon, t \geq T^{\prime}$. Without loss of generality, we may assume that $0<x(t)<\epsilon$ and $0<y(t)<\epsilon$ for all $t \geq 0$; then from system (1) we obtain

$$
-m z(t) \leq \frac{d z(t)}{d t} \leq(-m+\gamma \epsilon) z(t), \quad \gamma=\frac{e_{2} a_{2}}{b_{2}}+\frac{e_{3} a_{3}}{b_{3}} .
$$

From Lemmas 4 and 5, we have $v_{1}(t) \leq z(t) \leq v_{2}(t)$ and $v_{1}(t) \rightarrow z^{*}(t), v_{2}(t) \rightarrow v^{*}(t)$ as $t \rightarrow \infty$, where $v_{1}(t)$ and $v_{2}(t)$ are solutions of

$$
\begin{gathered}
\frac{d v_{1}(t)}{d t}=-m v_{1}(t), \quad t \neq n T, \quad t \neq(n+l-1) T \\
v_{1}\left(t^{+}\right)=\left(1-\delta_{3}\right) v_{1}(t), \quad t=(n+l-1) T \\
v_{1}\left(t^{+}\right)=v_{1}(t)+p, \quad t=n T, \\
v_{1}\left(0^{+}\right)=z\left(0^{+}\right), \\
\frac{d v_{2}(t)}{d t}=(-m+\gamma \epsilon) v_{2}(t), \quad t \neq n T, \quad t \neq(n+l-1) T, \\
v_{2}\left(t^{+}\right)=\left(1-\delta_{3}\right) v_{2}(t), \quad t=(n+l-1) T, \\
v_{2}\left(t^{+}\right)=v_{2}(t)+p, \quad t=n T \\
v_{2}\left(0^{+}\right)=z\left(0^{+}\right)
\end{gathered}
$$


respectively,

$$
v^{*}(t)=\left\{\begin{array}{c}
\frac{p \exp ((-m+\gamma \epsilon)(t-(n-1) T))}{1-\left(1-\delta_{3}\right) \exp ((-m+\gamma \epsilon) T)} \\
(n-1) T<t \leq(n+l-1) T, \\
\frac{p\left(1-\delta_{3}\right) \exp ((-m+\gamma \epsilon)(t-(n-1) T))}{1-\left(1-\delta_{3}\right) \exp ((-m+\gamma \epsilon) T)} \\
(n+l-1) T<t \leq n T .
\end{array}\right.
$$

Then, for any $\epsilon_{1}>0$ there exists a $T_{1}>0$ such that

$$
z^{*}(t)-\epsilon_{1}<z(t)<z^{*}(t)+\epsilon, \quad t>T_{1} .
$$

Let $\epsilon \rightarrow 0$; we have

$$
z^{*}(t)-\epsilon_{1}<z(t)<z^{*}(t)+\epsilon_{1}
$$

for $t$ large enough, which implies $z(t) \rightarrow z^{*}(t)$ as $t \rightarrow \infty$. This completes the proof.

Now we study the stability of the periodic solution, which explains the threshold expression of the release amount $p$ and the average harvest $\delta_{3}$ of the filter-feeding fish under the condition of all species persistence.

Theorem 8. The system (1) is permanent if

$$
\begin{aligned}
r_{1} T & -\frac{a_{2}}{b_{2} m} \frac{p}{1-\left(1-\delta_{3}\right) \exp (-m T)} \\
& \times\left(1-\delta_{3} \exp (-m l T)-\left(1-\delta_{3}\right) \exp (-m T)\right) \\
> & \ln \left(\frac{1}{1-\delta_{1}}\right), \\
r_{2} T & -\frac{a_{3}}{b_{3} m} \frac{p}{1-\left(1-\delta_{3}\right) \exp (-m T)} \\
& \times\left(1-\delta_{3} \exp (-m l T)-\left(1-\delta_{3}\right) \exp (-m T)\right) \\
> & \ln \left(\frac{1}{1-\delta_{2}}\right) .
\end{aligned}
$$

Proof. Suppose $X(t)=(x(t), y(t), z(t), p(t))$ is any solution of the system (1) with $X(0)>0$. From Lemma 6 , we assume that $x(t) \leq M, y(t) \leq M$, and $z(t) \leq M$ with $t \geq 0$. From.
(19), we have $z(t)>z^{*}(t)-\epsilon$ for all $t$ large enough and some $z(t) \geq p\left(1-\delta_{3}\right) \exp (-m T) /\left(1-\left(1-\delta_{3}\right) \exp (-m T)\right)-\epsilon \triangleq \zeta_{1}$ for $t$ large enough. Thus we only need to find $\zeta_{2}$ and $\zeta_{8}$ such that $x(t)>\zeta_{2}$ and $y(t)>\zeta_{8}$ for $t$ large enough. Now we will find a $\zeta_{2}$.

We will prove this in the following two steps. First, let $0<$ $\zeta_{4}, \epsilon_{1}>0$ be small enough such that

$$
\begin{aligned}
\eta_{1} \triangleq\left(1-\delta_{1}\right) \exp \left(\int_{(n+l-1) T}^{(n+l) T}\right. & \left(r_{1}\left(1-\frac{\zeta_{4}}{k_{1}}\right)-\frac{a_{1} M}{b_{1}}\right. \\
- & \left.\left.\frac{a_{2}}{b_{2}}\left(v_{3}^{*}(t)+\epsilon_{1}\right)\right) d t\right)>1 .
\end{aligned}
$$

Easy to prove that $x(t)<\zeta_{4}$ cannot hold all $t \geq 0$. Otherwise,

$$
\begin{gathered}
\frac{d z(t)}{d t} \leq-\left(m-\frac{e_{2} a_{2} x(t)}{b_{2}}-\frac{e_{3} a_{3} y(t)}{b_{3}}\right) z(t) \\
\leq-\left(m-\frac{e_{2} a_{2} \zeta_{4}}{b_{2}}-\frac{e_{3} a_{3} M}{b_{3}}\right) z(t), \\
t \neq n T, \quad t \neq(n+l-1) T, \\
z\left(t^{+}\right)=\left(1-\delta_{3}\right) z(t), \quad t=(n+l-1) T, \\
z\left(t^{+}\right)=z(t)+p, \quad t=n T, \\
z\left(0^{+}\right)=z_{0} .
\end{gathered}
$$

Then we have $z(t) \leq v_{3}(t)$ and $v_{3}(t) \rightarrow v_{3}^{*}(t)(t \rightarrow \infty)$, where $v_{3}(t)$ is the solution of

$$
\begin{gathered}
\frac{d v_{3}(t)}{d t}=-\left(m-\frac{e_{2} a_{2} \zeta_{4}}{b_{2}}-\frac{e_{3} a_{3} M}{b_{3}}\right) v_{3}(t), \\
t \neq n T, \quad t \neq(n+l-1) T, \\
v_{3}\left(t^{+}\right)=\left(1-\delta_{3}\right) v_{3}(t), \quad t=(n+l-1) T, \\
v_{3}\left(t^{+}\right)=v_{3}(t)+p, \quad t=n T, \\
v_{3}\left(0^{+}\right)=z_{0},
\end{gathered}
$$

$$
\begin{aligned}
& v_{3}^{*}(t) \\
& =\left\{\begin{array}{l}
\frac{p \exp \left(-\left(m-\left(e_{2} a_{2} \zeta_{4} / b_{2}\right)-\left(e_{3} a_{3} M / b_{3}\right)\right)(t-(n-1) T)\right)}{1-\left(1-\delta_{3}\right) \exp \left(-\left(m-\left(e_{2} a_{2} \zeta_{4} / b_{2}\right)-\left(e_{3} a_{3} M / b_{3}\right)\right) T\right)} \\
(n-1) T<t \leq(n+l-1) T \\
\frac{p\left(1-\delta_{3}\right) \exp \left(-\left(m-\left(e_{2} a_{2} \zeta_{4} / b_{2}\right)-\left(e_{3} a_{3} M / b_{3}\right)\right)(t-(n-1) T)\right)}{1-\left(1-\delta_{3}\right) \exp \left(-\left(m-\left(e_{2} a_{2} \zeta_{4} / b_{2}\right)-\left(e_{3} a_{3} M / b_{3}\right)\right) T\right)} \\
(n+l-1) T<t \leq n T .
\end{array}\right.
\end{aligned}
$$


Therefore, there exists a $T_{1}>0$ such that

$$
\begin{gathered}
z(t) \leq v_{3}(t)<v_{3}^{*}(t)+\epsilon_{1}, \\
\frac{d x(t)}{d t} \geq\left(r_{1}\left(1-\frac{\zeta_{4}}{k_{1}}\right)-\frac{a_{1} M}{b_{1}}-\frac{a_{2}}{b_{2}}\left(v_{3}^{*}(t)+\epsilon_{1}\right)\right) x(t), \\
t \neq n T, \quad t \neq(n+l-1) T, \\
x\left(t^{+}\right)=\left(1-\delta_{1}\right) x(t), \quad t=(n+l-1) T
\end{gathered}
$$

for $t>T_{1}$. Let $N_{1} \in N$ and $\left(N_{1}+l-1\right) T \geq T_{1}$. Integrating (35) on $((n+l-1) T,(n+l) T], n>N_{1}$, we can get

$$
\begin{gathered}
x((n+l) T) \geq x\left((n+l-1) T^{+}\right) \\
\quad \times \exp \left(\int _ { ( n + l - 1 ) T } ^ { ( n + l ) T } \left(r_{1}\left(1-\frac{\zeta_{4}}{k_{1}}\right)-\frac{a_{1} M}{b_{1}}\right.\right. \\
\left.\left.\quad-\frac{a_{2}}{b_{2}}\left(v_{3}^{*}(t)+\epsilon_{1}\right)\right) d t\right) \\
=x((n+l-1) T)\left(1-\delta_{1}\right) \\
\quad \times \exp \left(\int _ { ( n + l - 1 ) T } ^ { ( n + l ) T } \left(r_{1}\left(1-\frac{\zeta_{4}}{k_{1}}\right)-\frac{a_{1} M}{b_{1}}\right.\right. \\
\left.\left.\quad-\frac{a_{2}}{b_{2}}\left(v_{3}^{*}(t)+\epsilon_{1}\right)\right) d t\right) \\
=x((n+l-1) T) \eta_{1} .
\end{gathered}
$$

Then $x\left(\left(N_{1}+n+l\right)\right) T \geq X\left(\left(N_{1}+l\right) T\right) \eta_{1}^{n} \rightarrow \infty$ as $n \rightarrow$ $\infty$, which is a contradiction to the boundedness of $x(t)$. Hence there exists a $t_{1}>0$ such that $x\left(t_{1}\right) \geq \zeta_{4}$. So, there exists a $t_{3}>t_{1}$ such that $x\left(t_{3}\right) \geq \zeta_{4}$.

Second, if $x(t) \geq \zeta_{4}$ for all $t \geq t_{3}$, then our aim is obtained. Hence we only need to consider those solutions which leave the region $R=\left\{X(t) \in R_{+}^{3}: x(t) \leq \zeta_{2}, y(t) \leq\right.$ $\left.\zeta_{8}, z(t) \leq \zeta_{1}\right\}$ and reenter it again. Let $t^{*}=\inf _{t \geq t_{3}}\left\{x(t)<\zeta_{4}\right\}$, there are two possible cases for $t^{*}$.

Case I. Consider $t^{*}=\left(n_{1}+l-1\right) T, n_{1} \in N$. Then $x(t) \geq \zeta_{4}$ for $t \in\left[t_{3}, t^{*}\right]$ and $\left(1-\delta_{1}\right) \zeta_{4} \leq x\left(t^{*+}\right)=\left(1-\delta_{1}\right) x\left(t^{*}\right)<\zeta_{4}$. Select $n_{2}, n_{3} \in N$ such that

$$
\begin{aligned}
& \left(n_{2}-1\right) T>\frac{\ln \left(\epsilon_{1} /(M+p)\right)}{\left(-m+\left(e_{2} a_{2} \zeta_{4} / b_{2}\right)+\left(e_{3} a_{3} M / b_{3}\right)\right)}, \\
& \left(1-\delta_{1}\right)^{n_{2}} \exp \left(\left(n_{2} \eta_{2} T\right) \eta_{1}^{n_{3}}\right) \\
& >\left(1-\delta_{1}\right)^{n_{2}} \exp \left(\left(n_{2}+1\right) \eta_{2} T\right) \eta_{1}^{n_{3}}>1,
\end{aligned}
$$

where $\eta_{2}=\left(r_{1}\left(1-\left(\zeta_{4} / k_{1}\right)\right)-\left(a_{1} M / b_{1}\right)-\left(a_{2} M / b_{2}\right)\right)<0$. Let $\bar{T}=n_{2} T+n_{3} T$. We claim that there must be a $t_{4} \epsilon$ $\left(t^{*}, t^{*}+\bar{T}\right]$ such that $x(t)>\zeta_{4}$. Otherwise, consider (32) with $v_{3}\left(t^{*+}\right)=z\left(t^{*+}\right)$, and we have

$$
v_{3}(t)=\left\{\begin{array}{l}
\left(1-\delta_{3}\right)^{n-\left(n_{1}+1\right)}\left(v_{3}\left(n_{1} T^{+}\right)-\frac{p}{1-\left(1-\delta_{3}\right) \exp \left(\left(-m+\left(e_{2} a_{2} \zeta_{4} / b_{2}\right)+\left(e_{3} a_{3} M / b_{3}\right)\right) T\right)}\right) \\
\quad \times \exp \left(\left(-m+\frac{e_{2} a_{2} \zeta_{4}}{b_{2}}+\frac{e_{3} a_{3} M}{b_{3}}\right)\left(t-n_{1} T\right)\right)+v_{3}^{*}(t), \\
\left(1-\delta_{3}\right)^{(n-1)}\left(v_{3}\left(n_{1} T^{+}\right)-\frac{(n-1) T<t \leq(n+l-1) T,}{1-\left(1-\delta_{3}\right) \exp \left(\left(-m+\left(e_{2} a_{2} \zeta_{4} / b_{2}\right)+\left(e_{3} a_{3} M / b_{3}\right)\right) T\right)}\right) \\
\quad \times \exp \left(\left(-m+\frac{e_{2} a_{2} \zeta_{4}}{b_{2}}+\frac{e_{3} a_{3} M}{b_{3}}\right)\left(t-n_{1} T\right)\right)+v_{3}^{*}(t), \\
\quad(n+l-1) T<t \leq n T
\end{array}\right.
$$

and $n_{1}+1 \leq n \leq n_{1}+n_{2}+n_{3}$. Then

$$
\begin{aligned}
& \left|v_{3}(t)-v_{3}^{*}(t)\right| \\
& <(M+p) \exp \left(\left(-m_{+} \frac{e_{2} a_{2} \zeta_{4}}{b_{2}}+\frac{e_{3} a_{3} M}{b_{3}}\right)\left(t-n_{1} T\right)\right) \\
& <\epsilon_{1}, \quad z(t) \leq v_{3}(t) \leq V_{3}^{*}(t)+\epsilon_{1},
\end{aligned}
$$

$\left(n_{1}+n_{2}+n_{3}\right) T \leq t \leq t^{*}+\bar{T}$, which implies that (35) holds for $t^{*}+n_{2} T \leq t \leq t^{*}+\bar{T}$. As in the first step, we have

$$
x\left(t^{*}+n_{2} T\right) \geq x\left(t^{*}+n_{2} T\right) \eta_{1}^{n_{3}} .
$$

The first equation of the system (1) gives

$$
\begin{gathered}
\frac{d x(t)}{d t} \geq\left(r_{1}\left(1-\frac{\zeta_{4}}{k}\right)-\frac{a_{1} M}{b_{1}}-\frac{a_{2} M}{b_{2}}\right) x(t), \\
t \neq n T, \quad t \neq(n+l-1) T, \\
x\left(t^{+}\right)=\left(1-\delta_{1}\right) x(t), \quad t=(n+l-1) T .
\end{gathered}
$$


Integrating (41) on $\left[t^{*}, t^{*}+n_{2} T\right]$, we have

$$
x\left(t^{*}+n_{2} T\right) \geq \zeta_{4}\left(1-\delta_{1}\right)^{n_{2}} \exp \left(n_{2} \eta_{2} T\right) .
$$

Thus $x\left(t^{*}+\bar{T}\right) \geq \zeta_{4}\left(1-\delta_{1}\right)^{n_{2}} \exp \left(n_{2} \eta_{2} T\right) \eta_{1}^{n_{3}}>\zeta_{4}$, and this is a contradiction. Let $\bar{t}=\inf _{t \geq t^{*}}\left\{x(t)>\zeta_{4}\right\}$, and then $x(t) \leq$ $\zeta_{4}, x(\bar{t})=\zeta_{4}$, for $t \in\left(t^{*}, \bar{t}\right)$; we have

$$
x(t) \geq \zeta_{4}\left(1-\delta_{1}\right)^{n_{2}+n_{3}} \exp \left(\left(n_{2}+n_{3}\right) \eta_{2} T\right) \triangleq \zeta_{5} .
$$

For $t>\bar{t}$, the same arguments can be continued since $x(\bar{t}) \geq \zeta_{4}$.

Case II. Consider $t^{*} \neq\left(n_{1}+l-1\right) T, n_{1} \in N$. Then $x(t) \geq \zeta_{4}$ for $t \in\left[t_{3}, t^{*}\right]$ and $x\left(t^{*}\right)=\zeta_{4}$, and suppose $t^{*} \in\left(\left(n_{1}^{\prime}+l-\right.\right.$ $\left.1) T,\left(n_{1}^{\prime}+l\right) T\right), n_{1}^{\prime} \in N$. There are two possible cases for $t \in$ $\left(t^{*},\left(n_{1}^{\prime}+l\right) T\right)$.

Case IIa. $\left(x(t) \leq \zeta_{4}\right.$ for all $\left.t \in\left(t^{*},\left(n_{1}^{\prime}+l\right) T\right)\right)$. Similar to Case I, we can prove that there must be a $t_{2}^{\prime} \in\left[\left(n_{1}^{\prime}+l\right) T,\left(n_{1}^{\prime}+l\right) T+\bar{T}\right]$ such that $x\left(t_{1}^{\prime}\right)>\zeta_{4}$. Here we omit it. Let $\widetilde{t}=\inf _{t \geq t^{*}}\{x(t)>$ $\left.\zeta_{4}\right\}$; then $x(\widetilde{t}) \leq \zeta_{4}$. For $t \in\left(t^{*}, \widetilde{t}\right)$, we have

$$
x(t) \geq \zeta_{4}\left(1-\delta_{1}\right)^{n_{2} n_{3}} \exp \left(\left(n_{2}+n_{3}+1\right) \eta_{2} T\right) .
$$

Let $\zeta_{6} \triangleq \zeta_{4}\left(1-\delta_{1}\right)^{n_{2} n_{3}} \exp \left(\left(n_{2}+n_{3}+1\right) \eta_{2} T\right)<\zeta_{5}$, so $x(t) \geq \zeta_{6}$ for $t \in\left(t^{*}, \widetilde{t}\right)$. For $t>\widetilde{t}$, the same arguments can be continued since $x(\widetilde{t}) \geq \zeta_{4}$.

Case IIb. There exists a $t \in\left(t^{*},\left(n_{1}^{\prime}+l\right) T\right)$ such that $x(t)>$ $\zeta_{4}$. Let $\tilde{t}=\inf _{t \geq t^{*}}\left\{x(t)>\zeta_{4}\right\}$; then $x(\widetilde{t}) \leq \zeta_{4}$ for $t \in$ $\left(t^{*}, \widetilde{t}\right)$ and $x(\widetilde{t})=\zeta_{4}$. For $t \in\left(t^{*}, \widetilde{t}\right)$, (41) holds true. Integrating (41) on $\left(t^{*}, \widetilde{t}\right)$, we have

$$
x(t) \geq x\left(t^{*}\right) \exp \left(\eta_{2}\left(t-t^{*}\right)\right) \geq \zeta_{4} \exp \left(\eta_{2} T\right)>\zeta_{6} .
$$

Since $x(t) \geq \zeta_{4}$ for $t>\tilde{t}$, the same arguments can be continued. Hence $x(t) \geq \zeta_{6}$ for all $t>\widetilde{t}$. The proved method for $y(t)$ is similar to the proved method for $x(t)$. Set $\Omega=$ $\left\{(x, y, x): x \geq \zeta_{2}, y \geq \zeta_{8}, z \geq \zeta_{1}, x+y+z \leq 3 M\right\}$. Obviously, we know that the set $\Omega \in$ int $R_{+}^{3}$ is global attractors. every solution of system (1) will eventually enter and remain in region $\Omega$. Therefore, system (1) is permanent. The proof is completed.

Using the theoretical analysis, we obtain the threshold expression of the release amount $p$ and the average harvest $\delta_{3}$ of the filter-feeding fish under the condition of some species extinction and all species persistence, which in turn provides a theoretical basis for the numerical simulation.

\section{Numerical Analysis}

The effective methods used for eutrophication removal, algal bloom control process, and associated public perception are a crucial consideration in developing effective systems of eutrophication removal management for Zeya reservoir; hence the sustainability analysis of the impulsive eutrophication controlling system must be investigated in detail. In order to study how to choose the value of the parameters $p$ and $\delta_{3}$ so that they can satisfy the actual monitoring data and enable Theorems 7 and 8 to be feasible, it is necessary to present a more in-depth analysis of the relationship among $p$ and $\delta_{3}$. Set $f_{1}\left(p, \delta_{3}\right)=r_{1} T-\left(a_{2} / b_{2} m\right)(p /(1-$ $\left.\left.\left(1-\delta_{3}\right) \exp (-m T)\right)\right)\left(1-\delta_{3} \exp (-m l T)-\left(1-\delta_{3}\right) \exp (-m T)\right)-$ $\ln \left(1 /\left(1-\delta_{1}\right)\right)$, and $f_{2}\left(p, \delta_{3}\right)=r_{2} T-\left(a_{3} / b_{3} m\right)(p /(1-(1-$ $\left.\left.\left.\delta_{3}\right) \exp (-m T)\right)\right)\left(1-\delta_{3} \exp (-m l T)-\left(1-\delta_{3}\right) \exp (-m T)\right)-$ $\ln \left(1 /\left(1-\delta_{2}\right)\right)$. On the basis of the actual monitoring data of the Zeya reservoir from year 2005 to year 2011 and laboratory controlling experiment data, the values of the parameters can be taken as follows: $r_{1}=0.75, r_{2}=0.8, k_{1}=20, k_{2}=10$, $a_{1}=0.3, a_{2}=0.35, a_{3}=0.5, e_{1}=0.85, e_{2}=0.5$, $e_{3}=0.45, b_{1}=2, b_{2}=2.2, b_{3}=2.5, m=0.15, \delta_{1}=$ $0.15, \delta_{2}=0.25, l=0.003$, and $T=30$. Hence we have $f_{1}\left(p, \delta_{3}\right)=-22.33748107+(1.060606061(0.9888910035-$ $\left.\left.0.9754817198 \delta_{3}\right) p\right) /\left(0.9888910035+0.01110899654 \delta_{3}\right)$ and $f_{2}\left(p, \delta_{3}\right)=-23.71231793+(1.333333333(0.9888910035-$ $\left.\left.0.9754817198 \delta_{3}\right) p\right) /\left(0.9888910035+0.01110899654 \delta_{3}\right)$. It is obvious to find from Figure 1(a) that there exist some values of $p$ and $\delta_{3}$ so that $f_{1}\left(p, \delta_{3}\right)<0$ and $f_{2}\left(p, \delta_{3}\right)<$ 0 or $f_{1}\left(p, \delta_{3}\right)>0$ and $f_{2}\left(p, \delta_{3}\right)>0$, which in turn confirms the existence of Theorems 7 and 8. From the actual stocking amount of the little filter-feeding fish in Zeya reservoir to control the algal bloom, we can choose biologically feasible parametric numerical value of $p$ as $p=35$. Further, it can be seen from Figure 1(b) that if the value of $\delta_{3}$ is less than 0.4 , there exist $f_{1}\left(p, \delta_{3}\right)>0$ and $f_{2}\left(p, \delta_{3}\right)>0$, but if the value of $\delta_{3}$ is greater than or equal to 0.4 , then the system (1) remains locally asymptotically stable. It is to be noted from Figure 2 that the time series solution of the filter-feeding fish population oscillates with stable limit cycle with increasing time. In contrast, both the algae population and the dominant zooplankton population rapidly decrease to their extinction with the increasing time. Meanwhile, these results further demonstrate that Theorems 7 and 8 are correct and feasible.

Considering the great uncertainty that still surrounds biological treatment mechanism, it is most prudent to interpret the simulation output on the basis of information and data obtained from observation and experiment. Simulation design offers tremendous flexibility to study the behavior dynamic of biological treatment process, so it is necessary to investigate that the release amount $p$ is how to affect the dynamics of biological treatment process with the parameter $\delta_{3}=0.4$ and $0<p \leq 35$. It is worth pointing out from Figure 3 that the system (1) with $0<p<35$ exhibits relatively complex dynamical behaviors: chaos, period-halving bifurcation, and periodic attractor, but the system (1) with $p=35$ remains locally asymptotically stable. Furthermore, it should be stressed that the dominant zooplankton population rapidly decreases to their extinction when the value of $p$ is greater than 4.4268; this phenomenon shows that the dominant zooplankton population will advance toward extinction with the increase of $p$. But it is regretful for this phenomenon because the dominant zooplankton population is one of the main food for the filter-feeding fish and can graze the algae population, and the idea of using biological eutrophication removal treatment to control the algal bloom not only can control the algal 


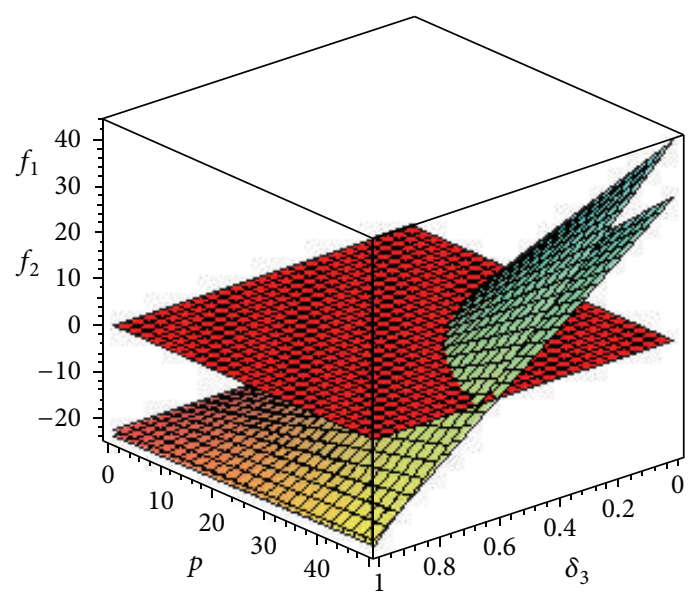

(a)

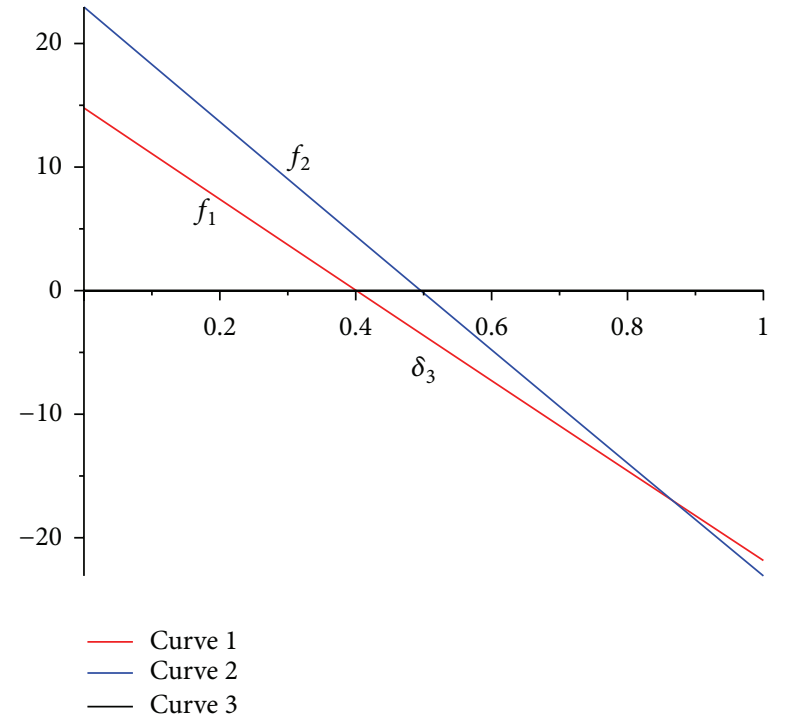

(b)

Figure 1: (a) The relationship diagram of the function $f_{1}\left(p, \delta_{3}\right)$ and the function $f_{2}\left(p, \delta_{3}\right)$ with respect to the parameters $p$ and $\delta_{3}$; (b) the relationship diagram of the function $f_{1}\left(p, \delta_{3}\right)$ and the function $f_{2}\left(p, \delta_{3}\right)$ with respect to the parameter $\delta_{3}$ with $p=35$.

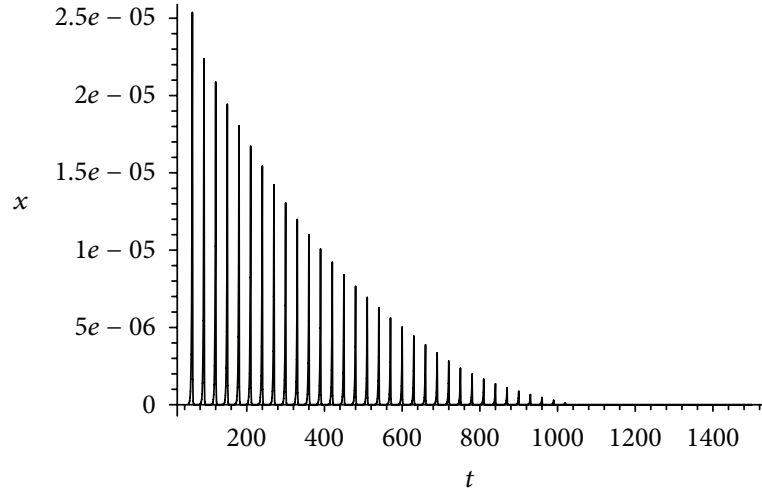

(a)

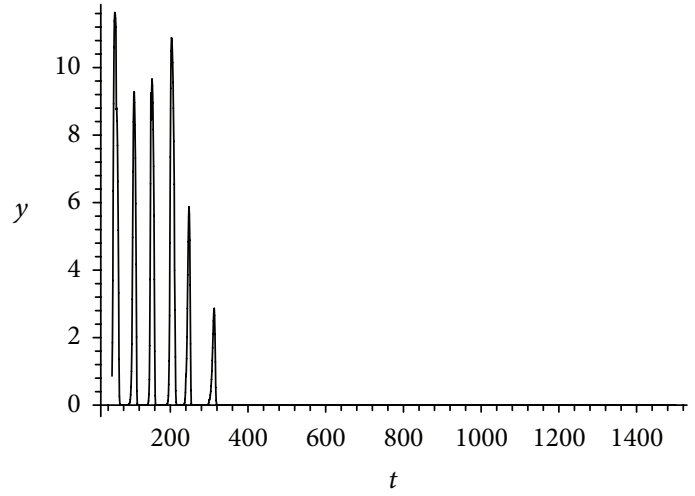

(b)

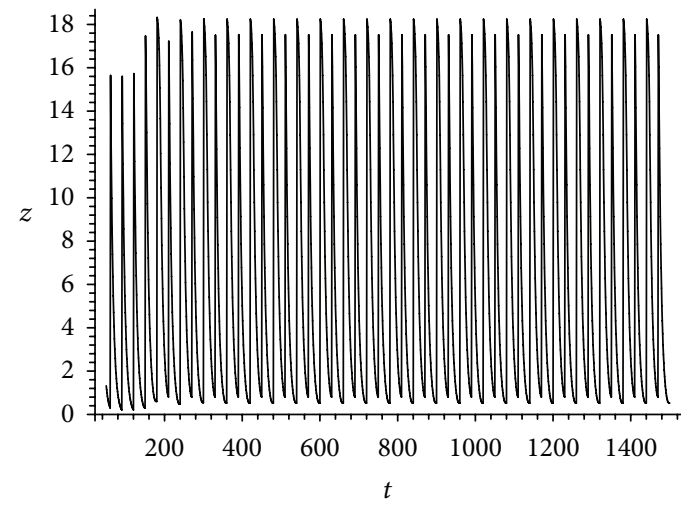

(c)

FIGURE 2: The dynamics of the system (1). 


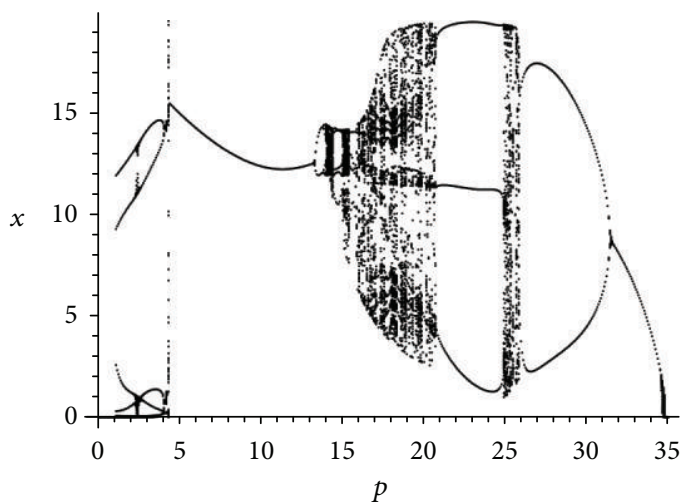

(a)

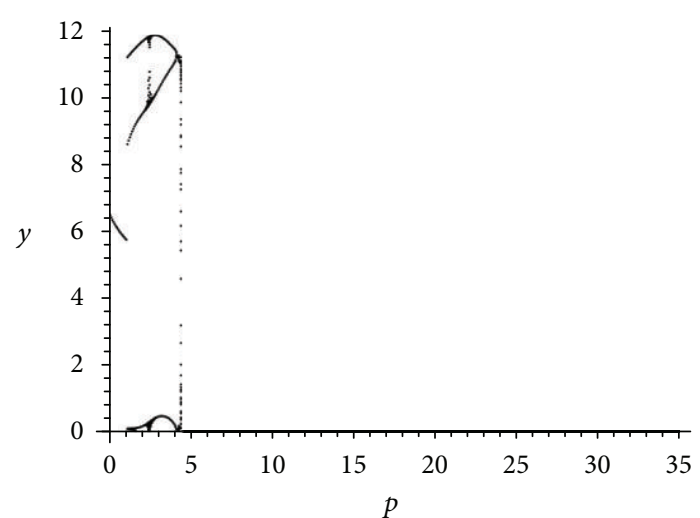

(b)

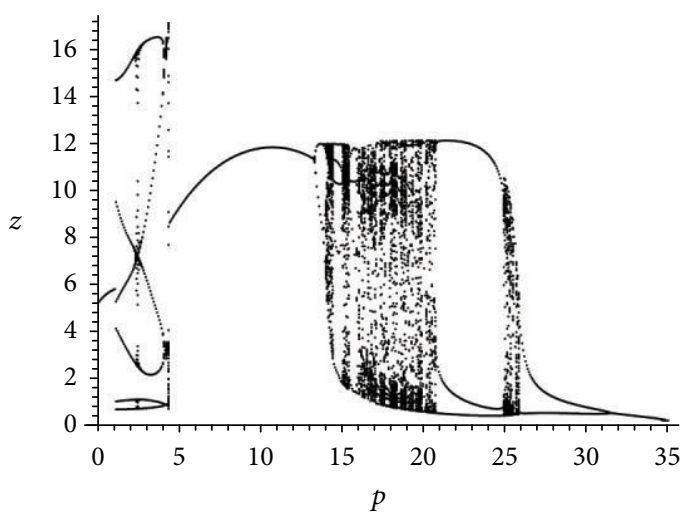

(c)

Figure 3: Bifurcation diagram of the system (1) with $0<p \leq 35$.

bloom, but also consider the sustainable development of eutrophication removal process with the maximizing economic benefits. Thus, controlling the desirable biomass of the dominant zooplankton population is a primary aim of operation and maintenance, and how to well carry out the eutrophication removal process is an extremely vital problem of Zeya reservoir.

In real ecosystem, the effect of some critical factors can be depicted by the properties and the dynamic behaviors of species [27, 28]. To more carefully investigate how to legitimately implement eutrophication removal treatment, Figure 4 depicts the long-run dynamics of system (1) for $0 \leq \delta_{3} \leq 1$ with $p=5$. It is easy to find from Figure 4 that the system exhibits relatively complex dynamical behaviors: chaos, period-halving bifurcation, and periodic attractor, but it is worth pointing out that, when the value of $\delta_{3}$ is beyond 0.6 , the algae population will rapidly go to extinction and the dominant zooplankton population will go toward a reasonable state, and the biomass of the dominant zooplankton population is so small that it does not cause the aggregation of the dominant zooplankton. Meanwhile, the biomass of the filter-feeding fish is feasible to maintain the sustainable development of eutrophication removal process with the maximizing economic benefits because of $p=5$. This result suggests that we can choose the value of $p$ and $\delta_{3}$ as 5 and 0.6 for legitimately implementing eutrophication removal treatment. For further analysis of the feasibility of this result, Figure 5 gives the dynamics of the system (1) with the increase of $t$ as well as $p=$ 5 and $\delta_{3}=0.6$. It is gratifying to see from Figure 5 that the algae population quickly goes to the extinct status with the increase of $t$, and the dominant zooplankton population is close to a reasonable number with the increase of $t$. It is worth encouraging that the filter-feeding fish can arrive to a viable amount so that the eutrophication removal process can be developed with the maximizing economic benefits. Therefore, it should be stressed that these results can perfectly confirm that the impulsive eutrophication controlling system can be well applied in the process of controlling the algal bloom in the theoretical work of eutrophication removal and mathematical model.

Based on the foregoing analysis, it is successful for impulsive eutrophication controlling system to implement the eutrophication removal process. The impulsive eutrophication controlling system not only can promote all wanted species persistence, but also is convenient for the manager to maintain the normal development of biological treatment process. Moreover, simulation analysis provides an approximation of the real biological controlling system behaviors; hence it is very easy to obtain the best value of $p$ and $\delta_{3}$, 


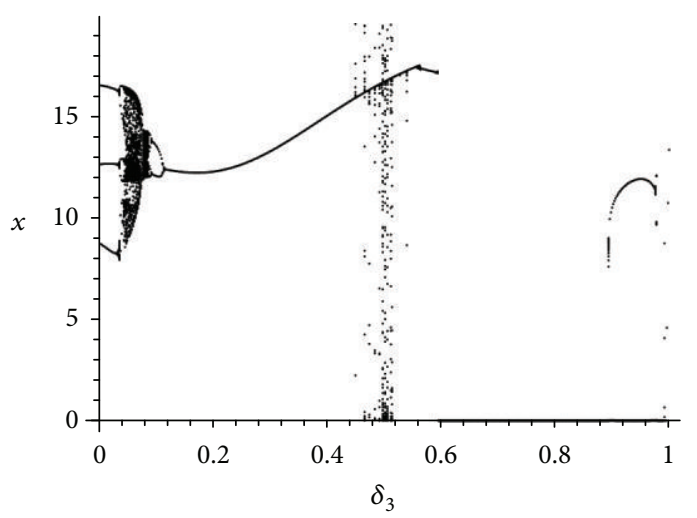

(a)

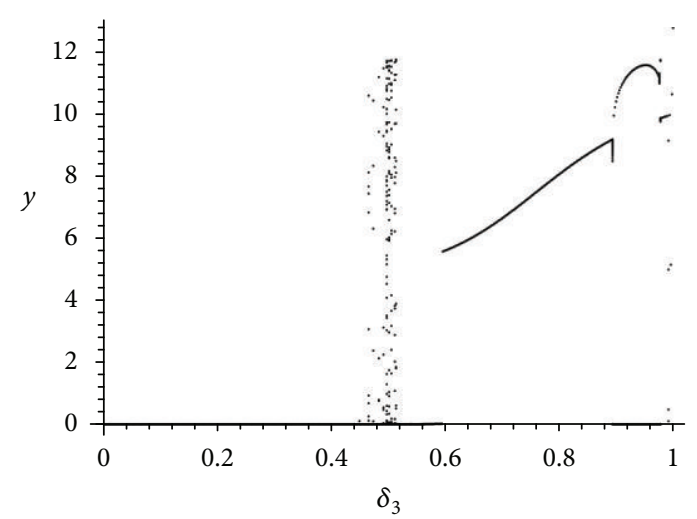

(b)

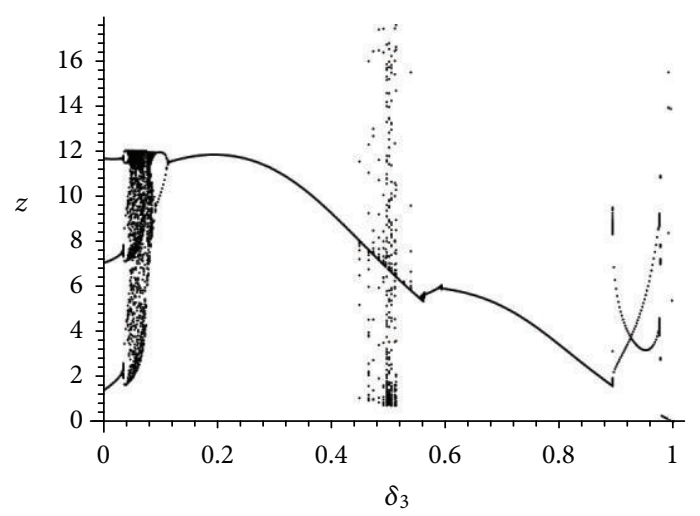

(c)

Figure 4: Bifurcation diagram of the system (1) with $0 \leq \delta_{3} \leq 1$.

which is in accordance with observations and in turn shows that the selection of the best value about some critical parameters in the view of population dynamics is feasible.

\section{Conclusions and Remarks}

The theoretical ecologists and applied mathematicians are now working on controlling the algal bloom in ecosystem, but the optimal way to eradicate the algae population to control the algal bloom is yet to know, but in the context of population growth dynamics, how to implement impulsive control strategy to prevent and control the algal bloom with the maximizing economic benefits is worthy of studying. In the paper, we take an approach to study how to better implement impulsive eutrophication controlling system to control the algal bloom and maintain the normal development of biological treatment process with maximizing economic benefits by using mathematical analysis and simulating the dynamics. On the basis of eutrophication ecology and differential equation, an impulsive eutrophication controlling system is studied analytically and numerically. A key advantage of the impulsive eutrophication controlling system is that it can be quite accurate to describe the interaction effect of some critical factors (fishermen catch and releasing small fry, etc.), which enables a systematic and logical procedure for fitting eutrophication mathematical system to real monitoring data and experiment data. mathematical theoretical works have been pursuing the investigation of two threshold functions $f_{1}\left(p, \delta_{3}\right)$ and $f_{2}\left(p, \delta_{3}\right)$ of two critical parameters $p$ and $\delta_{3}$, if $f_{1}\left(p, \delta_{3}\right)<0$ and $f_{2}\left(p, \delta_{3}\right)<$ 0 , the system (1) remain locally asymptotically stable, and if $f_{1}\left(p, \delta_{3}\right)>0$ and $f_{2}\left(p, \delta_{3}\right)>0$, the system (1) can ensure all species persistence. These results in turn provide a theoretical basis for the numerical simulation.

From the viewpoint of population dynamics, numerical simulation works have been pursuing the investigation of confirming the existence and feasibility of Theorems 7 and 8 and how to choose the best value of two critical parameters $p$ and $\delta_{3}$. Within this framework, it can be assumed that the sustainability of the impulsive eutrophication controlling system mainly depends on two critical parameters $p$ and $\delta_{3}$. Then the direct and indirect effects caused by critical parameters $p$ and $\delta_{3}$ are investigated by means of bifurcation analysis. These results indicate that if $p=5$ and $\delta_{3}=0.6$, the algae population will go to extinction, but the dominant zooplankton and the filterfeeding fish can survive in a feasible range, which can assure that the eutrophication removal process can be circulatingly developed with maximizing economic benefits. In addition, it is worthwhile to remark that mathematical theoretical results and numerical simulation results are complementary. It is 


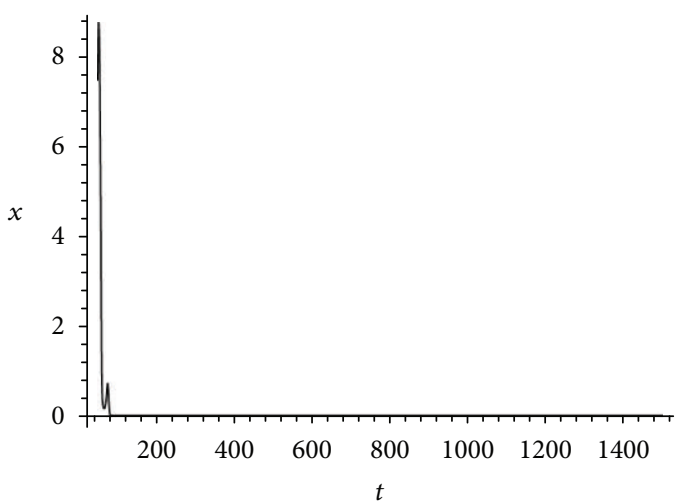

(a)

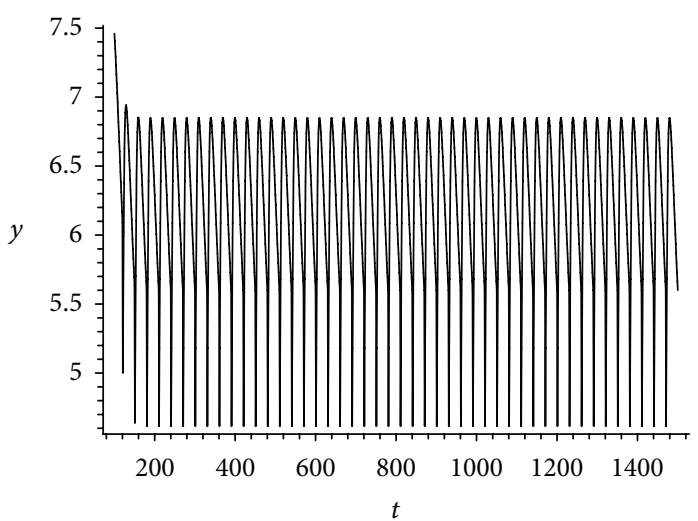

(b)

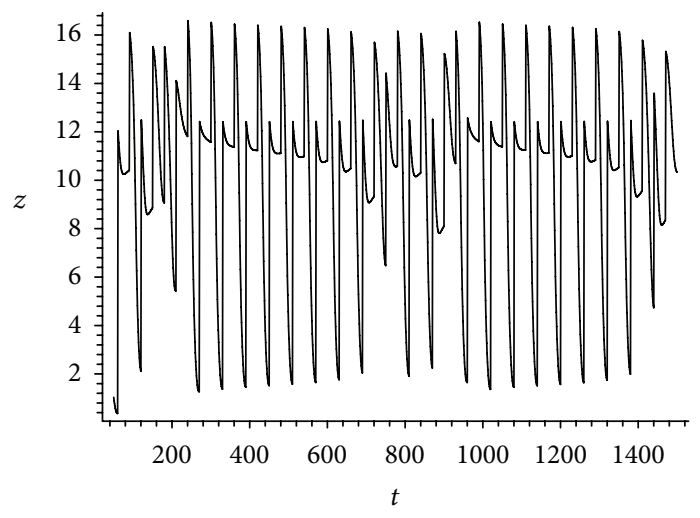

(c)

Figure 5: The dynamics of the system (1) with $p=5$ and $\delta_{3}=0.6$.

hopeful that these results have an important implication for eutrophication removal management; hence in the follow-up works, we will use the system to character the growth rule and the correlation between the algae population, the dominant zooplankton, and the filter-feeding fish.

\section{Acknowledgments}

This work was supported by the Zhejiang Provincial Natural Science Foundation of China (Grant no. LY13A010010), by the Key Program of Zhejiang Provincial Natural Science Foundation of China (Grant no. LZ12C03001), by the National Natural Science Foundation of China (Grant no. 31370381), by the National Key Basic Research Program of China (973 Program, Grant no. 2012CB426510) and by the Nonprofit Technology Research Projects of Zhejiang Province (Grant no. 2011C23119).

\section{References}

[1] S. Ryding and W. Rast, The Control of Eutrophication of Lakes and Reservoirs, The Parthenon Publishing Group, 1989.

[2] B. Qin, "Lake eutrophication: control countermeasures and recycling exploitation," Ecological Engineering, vol. 35, no. 11, pp. 1569-1573, 2009.
[3] M. Albay, R. Akcaalan, H. Tufekci, J. S. Metcalf, K. A. Beattie, and G. A. Codd, "Depth profiles of cyanobacterial hepatotoxins (microcystins) in three Turkish freshwater lakes," Hydrobiologia, vol. 505, pp. 89-95, 2003.

[4] D. Pyo and J. Jin, "Production and degradation of cyanobacterial toxin in water reservoir, Lake Soyang," Bulletin of the Korean Chemical Society, vol. 28, no. 5, pp. 800-804, 2007.

[5] G. Sun and L. Sheng, "Ecological engineering for eutrophication control in lake," Chinese Journal of Applied Ecology, vol. 12, no. 4, pp. 590-592, 2001.

[6] C. Alonso-Andicoberry, L. García-Villada, V. Lopez-Rodas, and E. Costas, "Catastrophic mortality of flamingos in a Spanish national park caused by cyanobacteria," Veterinary Record, vol. 151, no. 23, pp. 706-707, 2002.

[7] P. R. Hawkins, M. T. C. Runnegar, A. R. B. Jackson, and I. R. Falconer, "Severe hepatotoxicity caused by the tropical cyanobacterium (blue-green alga) Cylindrospermopsis raciborskii (Woloszynska) Seenaya and Subba Raju isolated from a domestic water supply reservoir," Applied and Environmental Microbiology, vol. 50, no. 5, pp. 1292-1295, 1985.

[8] A. Kouzminov, J. Ruck, and S. A. Wood, "New Zealand risk management approach for toxic cyanobacteria in drinking water," Australian and New Zealand Journal of Public Health, vol. 31, no. 3, pp. 275-281, 2007.

[9] G. A. Codd, "Cyanobacterial toxins, the perception of water quality, and the prioritisation of eutrophication control," Ecological Engineering, vol. 16, no. 1, pp. 51-60, 2000. 
[10] L. V. Bertalanffy, Theoretische Biologise, Bertalanffy, Berlin, Germany, 1932.

[11] H. Malchow, S. Petrovskii, and A. Medvinsky, "Pattern formation in models of plankton dynamics: a synthesis," Oceanologica Acta, vol. 24, no. 5, pp. 479-487, 2001.

[12] R. Kalčeva, J. Outrata, Z. Schindler, and M. Straškraba, "An optimization model for the economic control of reservoir eutrophication," Ecological Modelling, vol. 17, no. 2, pp. 121-128, 1982.

[13] J. M. Malmaeus and L. Håkanson, "Development of a Lake Eutrophication model," Ecological Modelling, vol. 171, no. 1-2, pp. 35-63, 2004.

[14] S. Chen, X. Chen, Y. Peng, and K. Peng, "A mathematical model of the effect of nitrogen and phosphorus on the growth of bluegreen algae population," Applied Mathematical Modelling, vol. 33, no. 2, pp. 1097-1106, 2009.

[15] C. G. Lee, T. D. Fletcher, and G. Z. Sun, "Nitrogen removal in constructed wetland systems," Engineering in Life Sciences, vol. 9, no. 1, pp. 11-22, 2009.

[16] P. Xie and J. Liu, "Practical success of biomanipulation using filter-feeding Fish to control cyanobacteria blooms: a synthesis of decades of research and application in a subtropical hypereutrophic lake," TheScientificWorldJournal, vol. 1, pp. 337-356, 2001.

[17] D. D. Bainov and P. S. Simeonov, Impulsive Differential Equations: Asymptotic Properties of the Solutions, World Scientific, Singapore, 1993.

[18] V. Lakshmikantham, D. D. Bainov, and P. C. Simeonov, Theory of Impulsive Differential Equations, World Scientific, Singapore, 1989.

[19] H. Yu, S. Zhong, R. P. Agarwal, and S. K. Sen, "Threespecies food web model with impulsive control strategy and chaos," Communications in Nonlinear Science and Numerical Simulation, vol. 16, no. 2, pp. 1002-1013, 2011.

[20] H. Yu, S. Zhong, R. P. Agarwal, and S. K. Sen, "Effect of seasonality on the dynamical behavior of an ecological system with impulsive control strategy," Journal of the Franklin Institute, vol. 348, no. 4, pp. 652-670, 2011.

[21] H. Yu, S. Zhong, and R. P. Agarwal, "Mathematics analysis and chaos in an ecological model with an impulsive control strategy," Communications in Nonlinear Science and Numerical Simulation, vol. 16, no. 2, pp. 776-786, 2011.

[22] X. M. Fan, Z. G. Wang, and F. Q. Jiang, "Dynamics of Mutualism-Competition-Predator system with BeddingtonDeAngelis functional responses and impulsive perturbations," Abstract and Applied Analysis, vol. 2012, Article ID 963486, 33 pages, 2012.

[23] G. Yu and H. Lu, "Permanence and almost periodic solutions of a discrete ratio-dependent leslie system with time delays and feedback controls," Abstract and Applied Analysis, vol. 2012, Article ID 358594, 31 pages, 2012.

[24] Y. Shen, F. Zhang, and K. Wang, "Permanence of a single species system with distributed time delay and feedback control," Abstract and Applied Analysis, vol. 2012, Article ID 867364, 14 pages, 2012.

[25] C. Lundberg, "Eutrophication, risk management and sustainability: the perceptions of different stakeholders in the northern Baltic Sea," Marine Pollution Bulletin, vol. 66, no. 1-2, pp. 143150, 2013.

[26] G. Perhar, G. B. Arhonditsis, and M. T. Brett, "Modeling zooplankton growth in lake washington: a mechanistic approach to physiology in a eutrophication model," Ecological Modelling, vol. 258, no. 10, pp. 101-121, 2013.

[27] F. F. Wu and X. Wang, "Eutrophication evaluation based on set pair analysis of baiyangdian lake, north china," Procedia Environmental Sciences, vol. 13, pp. 1030-1036, 2012.

[28] L. Wang, L. Liu, and B. Zheng, "Eutrophication development and its key regulating factors in a water-supply reservoir in north China," Journal of Environmental Sciences, vol. 25, no. 5, pp. 962-970, 2013. 


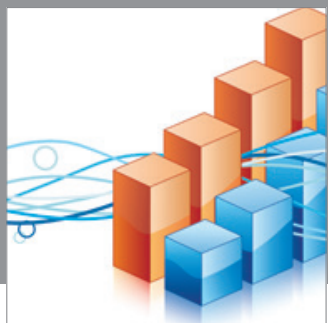

Advances in

Operations Research

mansans

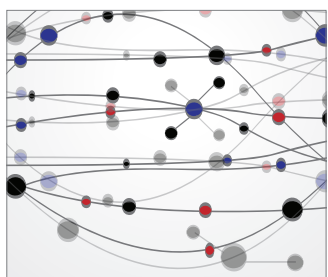

The Scientific World Journal
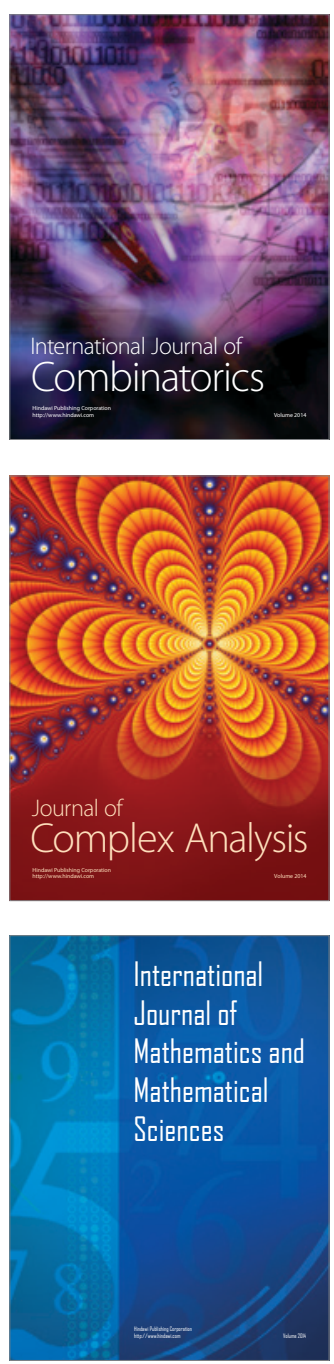
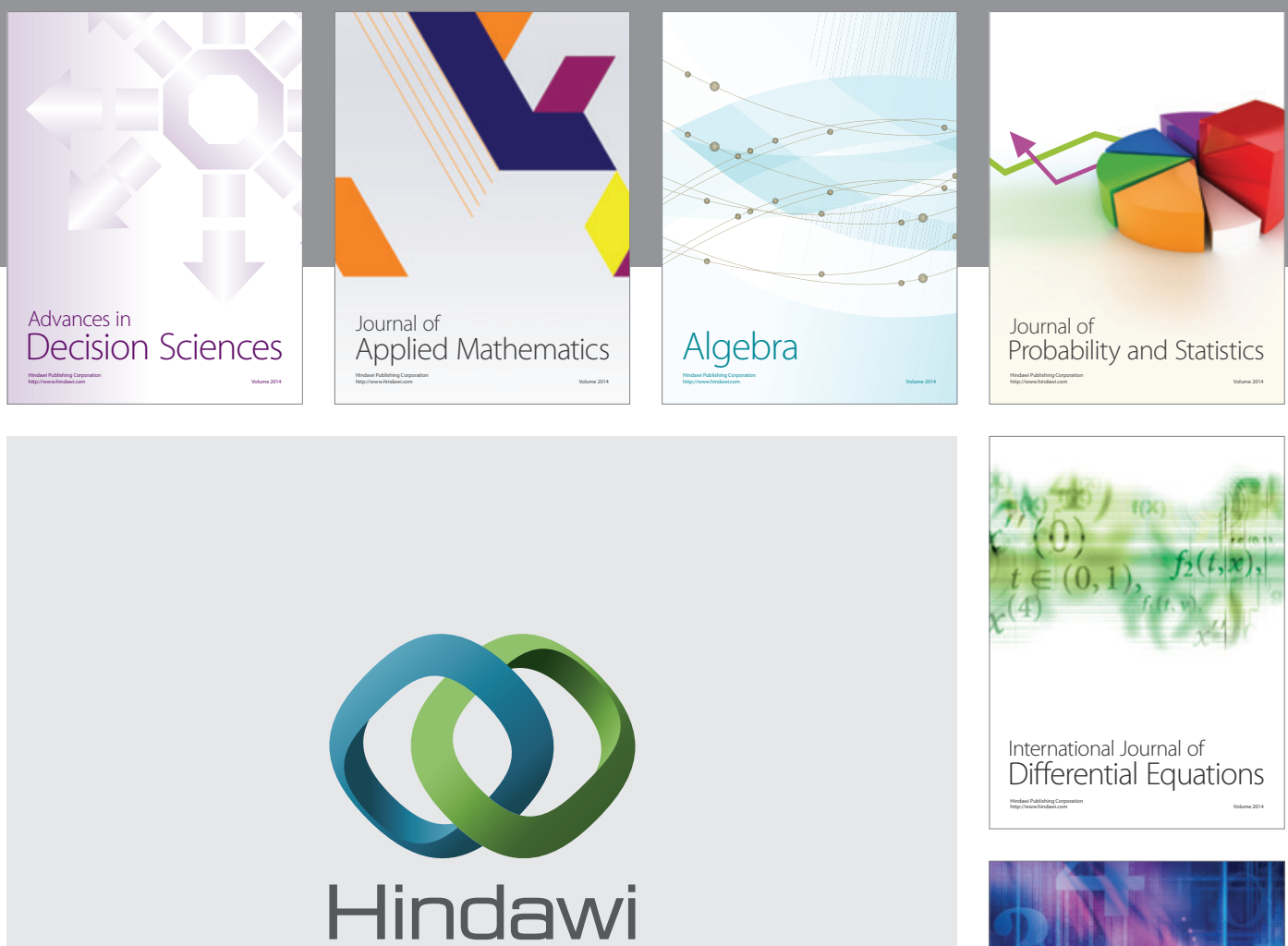

Submit your manuscripts at http://www.hindawi.com
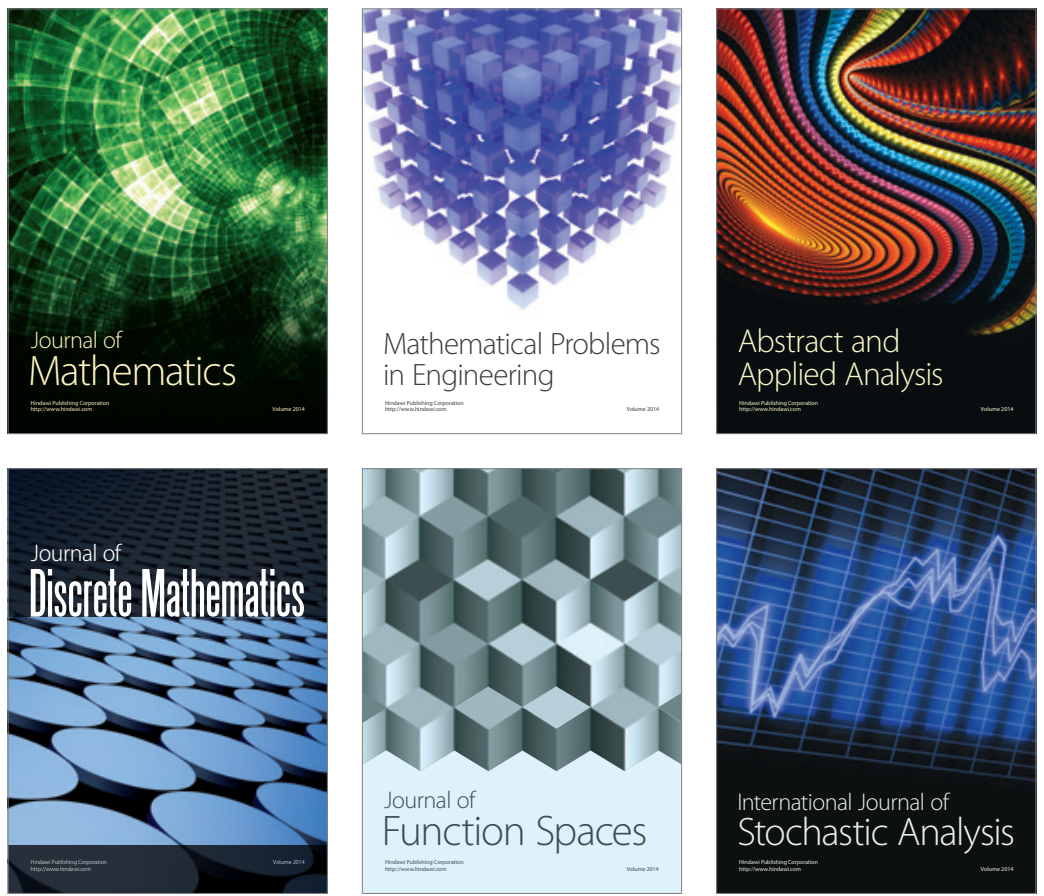

Journal of

Function Spaces

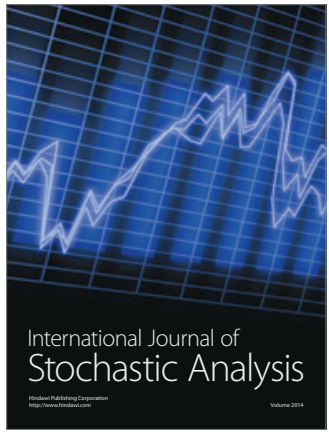

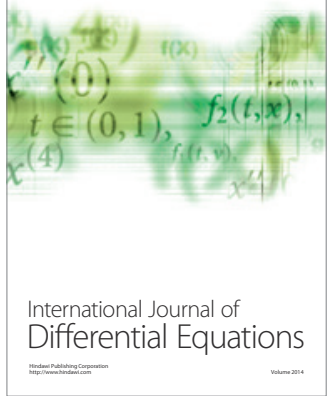
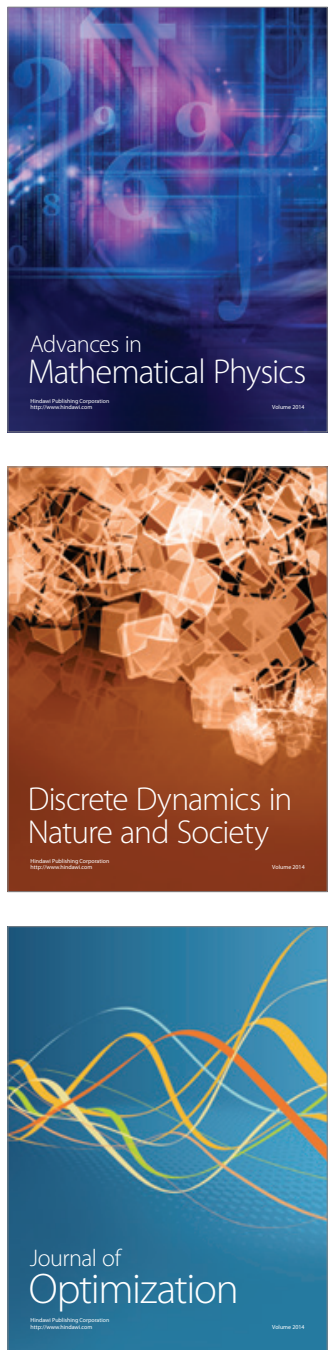